\title{
Recommendations for the nomenclature of cognitive
} change associated with anaesthesia and surgeryd 2018

L. Evered ${ }^{1,2, *}$ B. Silbert ${ }^{1,2}$, D. S. Knopman ${ }^{3}$, D. A. Scott ${ }^{1,2}$, S. T. DeKosky ${ }^{4}$, L. S. Rasmussen ${ }^{5}$, E. S. $\mathrm{Oh}^{6}$, G. Crosby ${ }^{7}$, M. Berger ${ }^{8}$, R. G. Eckenhoff ${ }^{9}$, and The Nomenclature Consensus Working Group ${ }^{\mathrm{a}}$

${ }^{1}$ St Vincent's Hospital, Melbourne, Fitzroy, Victoria, Australia, ${ }^{2}$ University of Melbourne, Fitzroy, Victoria, Australia, ${ }^{3}$ Department of Neurology, Mayo Clinic, Rochester, MN, USA, ${ }^{4}$ Department of Neurology,

McKnight Brain Institute, University of Florida, Gainesville, FL, USA, ${ }^{5}$ Department of Anaesthesia, Center of Head and Orthopaedics, Rigshospitalet, University of Copenhagen, Copenhagen, Denmark, ${ }^{6}$ Division of Geriatric Medicine and Gerontology, the Johns Hopkins University School of Medicine, Baltimore, MD, USA, ${ }^{7}$ Harvard Medical School, Brigham \& Women's Hospital, Boston, MA, USA, ${ }^{8}$ Neurologic Outcomes Research Group, Anesthesiology Department, Duke University Medical Center, Durham, NC, USA and ${ }^{9}$ Department of Anesthesiology and Critical Care, University of Pennsylvania School of Medicine, Philadelphia, PA, USA

*Corresponding author. E-mail: lis.evered@svha.org.au

${ }^{\text {a }}$ The Nomenclature Consensus Working Group participants and affiliations are listed in Appendix B section. 


\section{Abstract}

Cognitive change affecting patients after anaesthesia and surgery has been recognised for more than 100 yr. Research into cognitive change after anaesthesia and surgery accelerated in the 1980s when multiple studies utilised detailed neuropsychological testing for assessment of cognitive change after cardiac surgery. This body of work consistently documented decline in cognitive function in elderly patients after anaesthesia and surgery, and cognitive changes have been identified up to $7.5 \mathrm{yr}$ afterwards. Importantly, other studies have identified that the incidence of cognitive change is similar after non-cardiac surgery. Other than the inclusion of non-surgical control groups to calculate postoperative cognitive dysfunction, research into these cognitive changes in the perioperative period has been undertaken in isolation from cognitive studies in the general population. The aim of this work is to develop similar terminology to that used in cognitive classifications of the general population for use in investigations of cognitive changes after anaesthesia and surgery. A multispecialty working group followed a modified Delphi procedure with no prespecified number of rounds comprised of three face-to-face meetings followed by online editing of draft versions.

Two major classification guidelines [Diagnostic and Statistical Manual for Mental Disorders, fifth edition (DSM-5) and National Institute for Aging and the Alzheimer Association (NIA-AA)] are used outside of anaesthesia and surgery, and may be useful for inclusion of biomarkers in research. For clinical purposes, it is recommended to use the DSM-5 nomenclature. The working group recommends that 'perioperative neurocognitive disorders' be used as an overarching

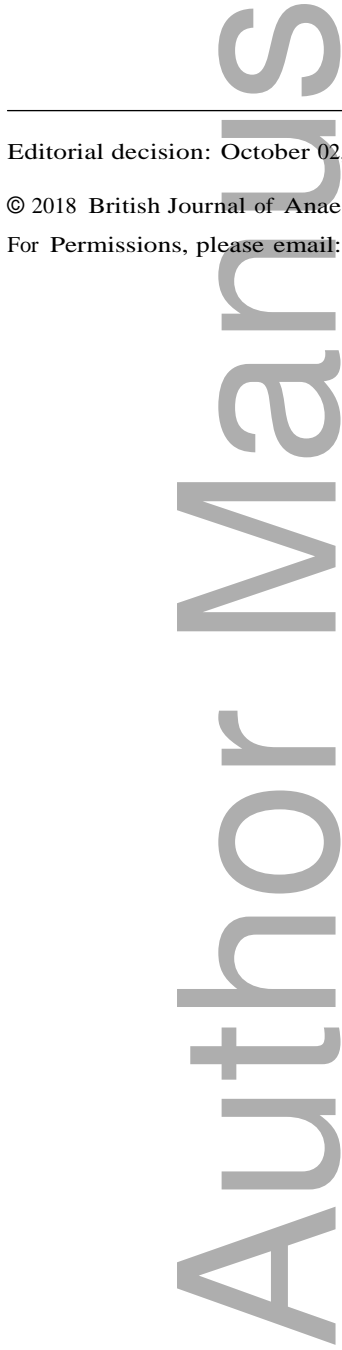


term for cognitive impairment identified in the preoperative or postoperative period. This includes cognitive decline diagnosed before operation (described as neurocognitive disorder); any form of acute event (postoperative delirium) and cognitive decline diagnosed up to 30 days after the procedure (delayed neurocognitive recovery) and up to 12 months (postoperative neurocognitive disorder).

Keywords: cognition disorders; delirium; neurocognitive disorders; postoperative complications

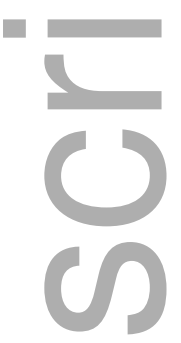

Cognitive change affecting patients after anaesthesia and surgery, particularly in the elderly, has been recognised in one form or another for more than $100 \mathrm{yr}$. Many clinicians are familiar with the clinical syndrome of delirium, a set of fluctuating changes in attention, mental status, and level of consciousness, which is often seen after anaesthesia and surgery. Aside from delirium, a large body of research has examined postoperative cognitive dysfunction (POCD) or decline after full recovery of consciousness, and persisting well beyond the expected pharmacological and physiological effects of anaesthetic drugs. Anaesthesia almost always accompanies surgery and its associated stresses, including healing and inflammation, and therefore the two will be considered together in this document.

Clinical complaints prompted research into cognitive change after anaesthesia and surgery, which accelerated in the 1980s when multiple studies used detailed neuropsychological testing for assessment after cardiac surgery. This body of work consistently documented decline in cognitive function in elderly patients in the short (7 days) and medium (1e3 months) ${ }^{1 \text { e3 }}$ term after anaesthesia and surgery, even in the absence of symptoms. The natural history of these changes still requires further investigation, and although cognitive changes have been identified as long as $7.5 \mathrm{yr}$ afterwards, ${ }^{4}$ causal associations remain unclear at least in part because of lack of well-defined baseline status, lack of control groups in most studies, and other methodological limitations of previous work. Consistently, studies have confirmed that cognitive decline is associated with increasing age, lower premorbid intelligence quotient, fewer years of education, or a combination of these. ${ }^{5}$ Further understanding has been complicated by significant heterogeneity in the type and number of tests administered, the criteria or definition for change, and the timing of administration of the tests. ${ }^{6}$ Details of these studies and the associated issues are provided in Supplement 1.

Interestingly, research into perioperative cognitive change has occurred in isolation from cognitive studies in the general population, and in other medical disciplines. In fact, periop- erative cognition has become largely a research area rather than a clinical state; subjective complaints are rarely sought or reported and capacity for activities of daily living (ADLs) is overlooked. Elderly individuals are a large subset of the general population, and, when normalised to population, are the 
represent $<14 \%$ of the population. By 2050, they will comprise $25 \%$ of the population and receive $50 \%$ of all anaesthetics. ${ }^{7}$ In the USA, more than 19 million anaesthetics are administered to those aged $>65 \mathrm{yr}$ every year, and a similar increase in exposure is expected. ${ }^{8}$

2. The diagnostic criteria for the cognitive changes associated with anaesthesia and surgery detected by psychometric testing should not be differentiated from neurocognitive

diserders (NCD) in the general population, and should
therefore be aligned with the clinical diagnostic criteria of neurocognitive disorders such as those already used in the Diagnostic and Statistical Manual for Mental Disorders, fifth edition (DSM-5). ${ }^{9}$

3. The terminology of cognitive change associated with anaesthesia and surgery should be aligned with other NCD to promote cross-specialty communication, aid clinical management of patients, and further high-quality research.

4. Neurocognitive disorders occur frequently in the community with $14 \mathrm{e} 48 \%$ aged $>70$ yr suffering mild cognitive impairment $(\mathrm{MCI})^{10}$ and an additional $10 \%$ suffering dementia. ${ }^{11}$ Therefore many individuals will have these disorders, even if preclinical, before they undergo anaesthesia major consumers of operative care; yet for those with spontaneous postoperative cognitive complaints, there exists no nomenclature, diagnostic framework, or referral recommendations within the specialties of anaesthesiology and surgery.

We consider a formal classification to be critical at this time for the following reasons:

1. The number of patients aged $>60 \mathrm{yr}$ undergoing anaesthesia and surgery has increased significantly and is projected to increase further. In Australia, individuals $>60$ yr receive nearly one-third of all anaesthetics, although they

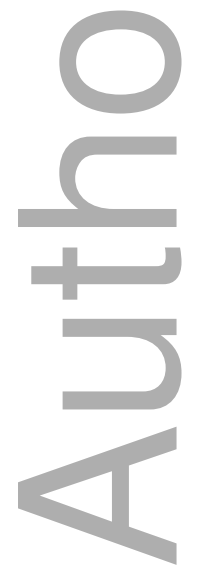

and surgery. Switching to a different terminology to classify these individuals when they are having an operation is confusing, counterintuitive, and counterproductive.

This work aims: 1) to develop and encourage the use of nomenclature and diagnostic criteria that are consistent with the terminology used in the wider clinical community when assessing and reporting cognitive impairment, but that retains the temporal association with anaesthesia and surgery; and 2) to align perioperative cognitive disorders with terms used in the community, namely the DSM-5 and National Institute for Aging and the Alzheimer Association (NIA-AA) definitions. This clinical nomenclature will offer a framework for understanding the impact of anaesthesia and surgery on outcomes, care, and management for the elderly and thereby enhance consistency of communication and reporting.

\section{Methods}

The working group comprised a multispecialty group of ex-

perts according to recommended Delphi procedures. This group was made up of invited international scientists and physicians, representing anaesthesiology, neurology, geriatrics, psychiatry, neuropsychology, surgery, and psychology [the Perioperative Cognition Nomenclature Working Group (listed below), hereafter, the 'working group']. The working group was assembled on three occasions to assess: 1) the current situation; 2) if perioperative cognitive disorders were sufficiently different from geriatric cognitive disorders to justify unique nomenclature; 3) how to include unique features of the perioperative period; 4) how to achieve 
endorsement of the varied stake-holders; and 5) how to disseminate the final product. The working group broadly used the Delphi approach with no prespecified number of rounds, with discussion, consensus development, voting of terms, or both, during the face-to-face meetings. The first Delphi questionnaire was developed using a focus group (the first face-toface meeting held in Copenhagen, 2014) and a literature review (see Supplement 1). ${ }^{12}$ After a further two full-day meetings in Honolulu, HI, USA (March 2015) and Washington, DC, USA (July 2015), a draft document was prepared and disseminated widely via email to allow adequate commentary. Feedback was provided by $25 / 44$ (57\%) members for the second Delphi round (December 2015) and 21/41 (51\%) for the third Delphi round (September 2016), with many additional authors requesting no specifie changes. The final version was then distributed to all members who were required to reply in support or otherwise of the final document. Some further changes were made according to consensus at this final stage. This was then submitted to a multidisciplinary collection of journals for consideration of joint, simultaneous publication. Where disagreement arose, this was put to the group and a compromise obtained if possible. If no compromise was possible (one instance by one individual at final draft stage, October 2016), consensus was taken as the majority opinion of the group. Commentary will, no doubt, continue, and this nomenclature will be likely to evolve, as has been the case for the NIA-AA nomenclature. ${ }^{13,14}$

This terminology applies the rubric used by the American Psychiatric Association, whereby a multidisciplinary team work to produce a document clearly describing a nomenclature for use by various disciplines with multiple needs to communicate characteristics of mental disorders, ${ }^{9}$ thus enabling crossdisciplinary conversations, investigation of possible mechanisms across multiple medical disciplines and aligned interventions and outcomes, and applies to all adult patients undergoing anaesthesia and surgery. The publication of the most recent Diagnostic and Statistical Manual for Mental Disorders, fifth edition ${ }^{9}$ (DSM-5) presents an opportunity to align cognitive change detected after anaesthesia and surgery with cognitive change in other disciplines in terms of the criteria for classification, the definition of decline, impairment, or both, meaningful clinical interpretation, and clinical nomenclature, although at this time postoperative is not a specifier endorsed by the DSM-5. Supporting documents are provided as Supplement 1 (POCD background) and Supplement 2 (excerpts from DSM-5). An alternative nomenclature has been introduced by the NIA$\mathrm{AA},{ }^{13,14}$ which has also achieved widespread clinical usage and maps closely to the DSM-5 classifications. The working group elected to adopt the DSM-5 nomenclature for perioperative purposes, and acknowledge that these align with NIA-AA definitions. Guidance was provided by members of the working groups who participated in the DSM-5 and NIA-AA cognitive disorder nomenclature efforts. These recommendations are applicable to clinicians and researchers of all specialties involved in the care of older adults.

\section{Results}

Recommendations for perioperative NCD

We recommend that 'perioperative neurocognitive disorders' be used as an overarching term for cognitive impairment or change identified in the preoperative or postoperative period. This includes cognitive impairment diagnosed before 
operation (described below as NCD); any form of acute event (such as delirium; discussed below) and cognitive decline diagnosed up to 30 days after the procedure (as outlined below as delayed neurocognitive recovery) and up to 12 months (described below as NCD).

Recommendations for pre-existing cognitive

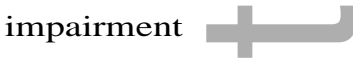

Pre-existing cognitive impairment is the term that has been used to refer to patients with objectively measurable subtle cognitive impairment at baseline (usually by comparison with population norms). As a preoperative assessment this measure of impairment cannot be related to the imminent anaesthesia and surgery and should be considered in terms of cognitive impairment that might be coincidentally identified in the community as mild NCD (MCI) or major NCD (dementia). See definitions in the section on recommendations for POCD.

\section{Recommendations for delirium}

'Postoperative delirium' should be recognised as a specific category consistent with DSM-5 terminology along with appropriate specifiers (substance intoxication, substance withdrawal, medication-induced, delirium because of other medical conditions, and delirium because of multiple aetiologies), once other specific causes have been excluded and the patient is in the immediate postoperative period.

The reported incidence of delirium in the elderly after anaesthesia and surgery is highly dependent on how it is diagnosed and screened, including how assessment staff are trained. After cardiac surgery, the incidence of delirium using chart review was $3 \%$, noted during routine clinical care was $8 \%$, using interviews with nurses was $9 \%$, and using daily mental status testing and application of a validated diagnostic algorithm was 53\%. ${ }^{15}$ The DSM-5 states that delirium occurs in $15 \mathrm{e} 3 \%$ of older individuals after operation and in $70 \mathrm{e} 87 \%$ of those in intensive care (page $600^{\circ}$ ). The term postoperative refers to a specific and known precipitating event (i.e. anaes-

thesia and surgery), which occurs annually in approximately one-third of individuals aged $>65 \mathrm{yr}$. Therefore, anaesthesia and surgery should be clearly identified as a potential precipitating cause of delirium. The term 'postoperative' would become a specifier for delirium occurring during the hospitalisation period after anaesthesia and surgery, in cases when other identifiable causes have been excluded. Implementation of this specifier is not only precise, but may facilitate therapeutic interventions.

We recognised that confusion between emergence delirium and postoperative delirium is possible. Nevertheless, after considerable debate it was agreed that a lucid period after emergence from anaesthesia should not be a requirement for the attribution of postoperative delirium. If the patient has a lucid interval this should be noted in the characterisation of that episode.

In summary, 'postoperative delirium' is defined as that which occurs in hospital up to 1 week postprocedure or until discharge (whichever occurs first) and meets DSM-5 diagnostic criteria. Other factors to consider when diagnosing postoperative delirium include:

1) Unmasked pre-existing vulnerabilities, for example, drugs/ alcohol/dementia/previous delirium

2) Persistent drug effects 
3) Other physiological perturbations including metabolic abnormalities, hypoxia, electrolyte imbalance, infection, etc.

4) Lucid interval (not mandatory, but should be documented)

Where delirium is observed during the hospitalisation phase and continues in the early post-discharge period (persistent delirium), the delirium should retain the postoperative specifier.

rifier.

Recommendations for POCD

POCD has been used in research studies to describe an objectively measurable decline in cognitive function at varying intervals after anaesthesia and surgery, up to 3 months e7.5 yr after surgery $4,16,17$ (Supplement 1). Generalisability to the clinical context needs to be carefully considered, as research has hitherto under-represented those with lower education, minority groups, and languages other than English, limiting translation to clinical practice.

Criteria for POCD have been confined to research and have typically rested on an objective decline in cognition as measured by neuropsychological tests, usually in the form of multiple tests administered as a test battery. In the absence of a control group, early investigations often defined individual patient decline as a postoperative decrement of 1 standard deviation (of the whole group at baseline) as a cutoff for decline in an individual test. In response to calls for appropriate control groups, ${ }^{18,19}$ investigators began to use controls to account for confounders (including time and learning effects). When control groups were used, the calculation of decline was often referenced to the expected change in the control group using one of several formulae for the reliable change index. ${ }^{20,21}$

In addition to different methods of calculating decline in an individual test and attributing overall decline, investigators have inadvertently introduced other sources of variation. These include variability in the number of tests administered, the number of tests required to decline to classify an individual as $\mathrm{POCD}$ positive, the statistical threshold to determine POCD, and the methods used to combine results into aggregate scores. ${ }^{6}$ Additionally, postoperative testing has been undertaken at variable time intervals after surgery. The end result of this heterogeneity is that there is no standardised definition of POCD, for either research or clinical purposes.

The research criteria used for POCD can be contrasted with the DSM-5 criteria for mild and major NCD. The DSM-5 stresses that both a cognitive concern by the individual, informant, or clinician and objective evidence is required to satisfy a diagnosis of NCD, and evidence of either maintained ADLs (mild NCD) or impaired ADLs (major NCD; Supplement
2). Thus, a major difference between POCD and NCD is the requirement for a cognitive concern in the latter and evidence of functional ability. The objective criteria for NCD are a decline of $1 \mathrm{e} 2$ standard deviations usually against a normative group for mild NCD and $>2$ standard deviations for major NCD. The value of NCD is that it includes clinical (subjective and functional) components, as do nearly all other DSM diagnoses, and thus aligns with standard clinical diagnostic criteria.

\section{Cognitive concern}

Taking an appropriate history including assessment of cognitive concerns both before and after anaesthesia and surgery is feasible and would align perioperative cognitive

\section{This article is protected by copyright. All rights reserved}


disorders with NCD. It also allows alignment for those patients spontaneously reporting cognitive symptoms after operation. Assessing symptoms before hospital discharge is often problematic. This period may be more complicated by postoperative delirium, mobility and ongoing acute pharmacological interventions (e.g. analgesics) than 'endogenous' cognitive issues per se. Even if at home, it is unlikely that they or an informant will be able to make an accurate assessment of subtle cognitive decline. Therefore, while alignment with NCD classification after discharge but before full recovery may be technically possible, the clinical relevance of this classification would be unclear, and hence we recommend a different term, 'delayed neurocognitive recovery'. Other terms, such as 'early NCD' were discussed, but were considered to be too similar to 'early MCI' or 'early Alzheimer's disease', labels that carry a considerably more ominous natural history than POCD detected or reported in this time window. While the term 'early' recognises that not all patients recover completely from this early form of POCD, studies indicate that many and perhaps most do, and thus we believe the default clinical diagnosis should indicate the potential for recovery. Should signs and symptoms of cognitive dysfunction persist past the expected time course for physical, physiological, and emotional recovery from surgery, then the term becomes postoperative mild or major NCD.

The time when this conversion of terms occurs will be 30 days after the procedure when recovery from all aspects of the surgery and hospitalisation should have occurred. This is consistent with hospital outcome data, and reflects that the acute effects of drugs, anaesthesia, pain, sleep, and nutritional hygiene, and the emotional stress of hospitalisation usually will have passed by 30 days. The incidence of delirium typically decreases by 30 days after surgery, although must still be excluded. ${ }^{13,19}$ Further, 28e30-day outcomes are commonly used as quality metrics for clinical performance. ${ }^{7}$ Criteria for decline should still be aligned with DSM-5 (1e2 standard deviations below controls/norms for mild NCD and 2 standard deviations below controls/norms for major NCD), but the use of 'delayed neurocognitive recovery' allows consideration of other factors at play.

It must be noted that high functioning individuals may not classify as impaired or declining using cognitive testing against norms, either on objective testing or on questionnaires (e.g. Clinicians Global Impression of Change ${ }^{22}$ ), which tend to be insensitive to subtle changes. This highlights the relevance of subjective reports of cognitive concerns. Therefore, a subjective report from the participant, informant or clinician is an essential element of diagnosing a perioperative neurocognitive disorder. However, because of the requirement for objective evidence of at least 1 standard deviation decline from norms to diagnose NCD, such high functioning individuals could be missed entirely despite the presence of symptoms. Thus, for clinical interpretation these need to be considered in the context of each individual.

Objective testing

Beyond early testing (up to 30 days), we recommend aligning the objective criteria of perioperative cognitive disorders with NCD as described in DSM-5 ${ }^{9}$ (Supplement 2 ). Mild NCD requires a decline, as compared with norms, of $1 \mathrm{e} 2$ standard deviations (or $3^{\text {rd }} \mathrm{e} 16^{\text {th }}$ percentile on tests that are not normally distributed). Major NCD requires a decline of $>2$ standard deviations

\section{This article is protected by copyright. All rights reserved}


(or $3^{\text {rd }}$ percentile or below if not normally distributed) in an appropriate neuropsychological assessment. Norms or controls must be used where assessment of change is not possible, and the above cut-points are acceptable. Decrements should be considered in terms of $\mathrm{z}$-scores calculated using norms or controls. Comparable age in the comparator group is always important. Norms may be general population data, or specific, appropriate control groups. Serial assessment of cognition is likely to be more robust that testing at a single time point. Thus, measurement of change via serial assessments is specifically mentioned in DSM-5 as a way to avoid problems of comparing high and low performers against norms. The current recommendations for objective criteria do not specify individual neuropsychological tests or the number of tests required in a battery, as such recommendations are not contained in DSM-5 or NIA-AA criteria. The objective criteria should be based according to the DSM-5 on 'evidence of modest (mild-NCD) or significant (major-NCD) cognitive decline from a previous level of performance in one or more cognitive domains (complex attention, executive function, learning and memory, language, perceptual-motor, or social cognition) ${ }^{\prime} .{ }^{9}$ These assessments refer to the use of psychometric assessments with the ability to objectively assess specific cognitive domains, not to the use of screening tools such as the Mini-mental State Examination or Montreal Cognitive Assessment.

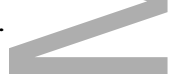

ADL assessment

Classification of mild and major NCD requires assessment of ADLs with an appropriate tool to measure subtle changes in function. For mild NCD evidence must be provided that daily function is overall maintained, whilst for major NCD evidence is required to confirm a decline in daily function from a previous level. This may be self-report or informantreport, and the changes may be subtle in nature. Assessment of instrumental activities of daily living is the most common form of assessment of subtle impairments in daily function.

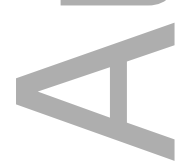

\section{Discussion}

Aligning nomenclature for POCD with NCD

As stated earlier, two major classification guidelines (DSM-5 and NIA-AA) are currently used more or less synonymously outside of the disciplines of anaesthesiology and surgery. Mild NCD and major NCD (DSM-5) map approximately onto $\mathrm{MCI}^{13}$ and dementia (NIA-AA), ${ }^{14}$ respectively, and could therefore be effectively used as interchangeable terms. The NIA-AA nomenclature makes provision for the inclusion of biomarkers, which is relevant for research purposes currently and perhaps for clinical purposes in the future. It is recommended to use only the DSM-5 nomenclature to avoid the potential negative connotations of the NIA-AA terms.

We therefore recommend that the clinical nomenclature for impairment in, or change in, cognition that is temporally associated with anaesthesia and surgery be changed from POCD to 'delayed neurocognitive recovery' and mild or major NCD (DSM-5), depending on timing and magnitude. Each will include appropriate specifiers and time-frames. DSM-5 advocates using a measure of change, but also allows diagnosis with reference to normative data. In the context of identifying changes after operation, the importance of defining baseline cognitive status is obvious.

Recommended nomenclature for perioperative NCDs

Please refer to Table 1:

Preoperative period: (in line with DSM-5 diagnosis undertaken independently of planned anaesthesia and surgery)

Mild NCD

Major NCD

After operation to the point immediately before the effects of anaesthesia and surgery are expected to have resolved (30 days):

Postoperative delirium

Table 1 Summary of perioperative cognitive disorder (POCD) nomenclature. NCD, neurocognitive disorders. ${ }^{*}$ The time point from a least 30 days when the effects of anaesthesia and surgery should have resolved. ${ }^{y}$ The postoperative specifier will be applied wher delirium is nersistent hevond hosnital discharge. ${ }^{z}$ Also annlies to assessments hefore medical readiness for discharge although it 


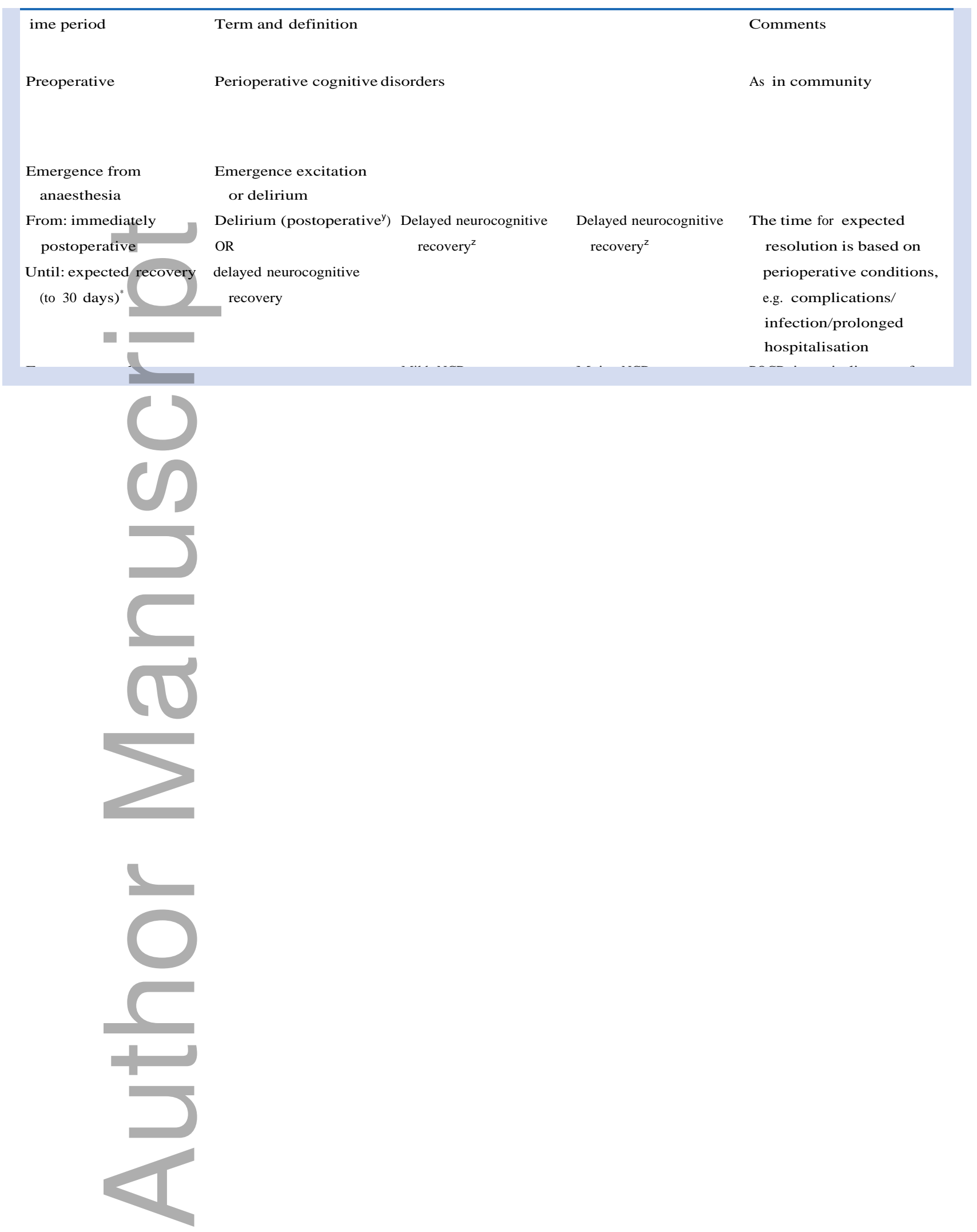


(postoperative specifier to be used if delirium is new or persistent in the perioperative period. If new postdischarge, it represents new delirium and will not attract the postoperative specifier)

Delayed neurocognitive recovery (definition is DSM-5 mild or major NCD)

This term to be used before 30 days. Assessments before medical readiness for discharge, because of the complexity of this period, should be recorded in the patient chart with appropriate description.

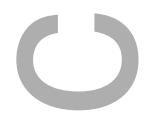

Expected recovery (30 days up to 12 months):

Postoperative mild neurocognitive disorder (POCD)

Postoperative major neurocognitive disorder (POCD)

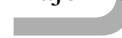

Up to 12 months will apply where new cognitive decline (new impairment or further decline) is not accounted for by any other medical condition. The 'postoperative (POCD)' specifier only applies beyond 12 months if the diagnosis (mild/major NCD) was made before 12 months. POCD is included as a calibrating specifier in parentheses for the transition period while integrating the new nomenclature.

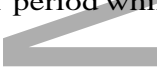

Concern has been raised that the specifier 'postoperative' implies a causal linkage to some aspect of anaesthesia and surgery. The term here is intended to only indicate a temporal relationship, and not aetiology. Acknowledging the temporal relationship will provide the opportunity for future research aligned with other age-related cognitive disorders to investigate possible aetiologies.

\section{Conclusions}

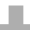

\section{.}

This proposal is to revise the nomenclature for perioperative cognitive disorders to align with DSM-5 criteria with appropriate specifiers and time-frame.

This new nomenclature for cognitive impairment before operation, or cognitive decline after operation, will conform to the three DSM-5 pillars of diagnosis for neurocognitive disorders:

1) Subjective complaint (participant, informant, clinician)

2) Objective impairment/change (mild: 1 e2 standard deviations below norms or controls; major: 2 standard deviations below norms or controls)

3) Instrumental ADLs (for major NCD/dementia a decline in function is required)
The one departure from DSM-5 nomenclature would be in the recovery period. Up to 30 days after the procedure, cognitive decline otherwise meeting DSM-5 criteria will be considered 'delayed neurocognitive recovery'. After this, the DSM-5 nomenclature will be adopted, but with temporal specifiers. 'Postoperative (POCD)' becomes a specifier similar to other specifiers in DSM-5, such as traumatic brain injury or substance abuse. As noted above, the term 'POCD' will be included in parentheses (at least in the short term) as a transitional calibrator: Thus the 'postoperative (POCD)' specifier will refer to a period from 30 days after anaesthesia and surgery to 12 months follow-up, using the DSM-5 diagnostic criteria:

'postoperative mild neurocognitive disorder (POCD)' and 'postoperative major neurocognitive disorder (POCD)'. 
This nomenclature will apply up to 12 months, assuming the cognitive decline cannot be accounted for by any other medical condition. After 12 months the 'postoperative (POCD)' specifier is no longer attached if first diagnosed after this time, as the aetiology cannot be reasonably linked to the prior surgery and anaesthesia episode. This routine DSM-5 nomenclature would also apply to preoperative assessments which have often been referred to as pre-existing cognitive impairment. ${ }^{23,24}$

\section{Authors' contributions}

Conception and design, manuscript preparation and critical revisions, final approval and agreement to be accountable for all aspects of the work:L.E., B.S., D.S.K., D.A.S., S.T.D.K., L.S.R., E.S.O., G.C., M.B., R.G.E.

All authors listed in the working group meet the full criteria for authorship.

\section{Declaration of interest}

The authors L.E., R.G.E., D.S.K., D.A.S., B.S., E.S.O., D.A., A.B., D.B., S.G.D., D.v.D., M.F.E., D.G., K.H., S.I., E.M., P.M., B.A.O., T.O., C.P., P.S., K.S., F.E.S., J.S., N.T., P.T., R.W., and Z.X. declare that they have no conflicts of interest.

M.B.: unrelated funding from Minnetronix Inc.; material support from Masimo; Associate Editor Journal of Alzheimer's Disease; participated in activities of the ASA BHI.

G.C.: Editor of Anesthesia \& Analgesia; Editor of Journal of Clinical Anesthesia.

S.T.D.K.: previously consulted for Roche, Novartis, Biogen, Acumen, Cognition Therapeutics, Amgen.

L.S.R.: editorial board member Acta Anaesthesiologica Scandinavica; wife received research funding and lecture fees from Merck.

L. E.: shareholder of Alphasyx Inc.

J.B.: payments and travel funding for consultancy from Claret Medical Inc.

M.M.: cofounded NeuroproteXeon, received founders' stock and stock options; previously consulted for Masimo.

C.L.: grant support from Forest, Glaxo-Smith-Kline, Eisai, Pfizer, Astra-Zenica, Lilly, Ortho-McNeil, Bristol-Myers, Novartis, NFL Benefits Office, Elan, Functional Neuromodulation. Consultant/Advisor for: Astra-Zenica, GlaxoSmith-Kline, Eisai, Novartis, Forest, Supernus, Adlyfe, Takeda, Wyeth, Lundbeck, Merz, Lilly, Pfizer, Genentach, Elan, NFL Players Association, NFL Benefits Office, Avamir, Zinfandel, BMS, Abvie, Janssen, Orion, Otsuka, Astellas.
Funding was provided for two of the face-to-face meetings by the Alzheimer's Association International Society to Advance Alzheimer's Research and Treatment (ISTAART), and the International Anesthesia Research Society (IARS).

\section{Appendix A. Supplementary data}

Supplementary data related to this article can be found at https://doi.org/10.1016/j.bja.2017.11.087.

\section{Appendix B}

The Nomenclature Consensus Working Group participants and affiliations:Lisbeth Evered MBiostat PhD St. Vincent's Hospital

\section{Funding}


Melbourne \& University of Melbourne, Australia, and Roderic G. Eckenhoff MD Department of Anesthesiology and Critical Care University of Pennsylvania School of Medicine Philadelphia, PA, USA, Co-Chairs; David Ames BA MD University of Melbourne Academic Unit for Psychiatry of Old Age, Melbourne Australia; Alex Bekker MD Department of Anesthesiology, Department of Physiology and Pharmacology, Rutgers New Jersey Medical School, Newark, NJ, USA; Miles Berger MD PhD Neurologic Outcomes Research Group, Anesthesiology Department, Duke University Medical Center, Durham, NC, USA; Deborah Blacker MD PhD Massachusetts General Hospital/Harvard Medical School, Harvard T.H. Chan School of Public Health, Boston, MA, USA; Jeffrey Browndyke PhD Department of Psychiatry \& Behavioral Sciences, Division of Geriatric Behavioral Health, Duke University Medical Center, Durham, NC, USA; Greg Crosby MD Harvard Medical School, Brigham \& Women's Hospital, Boston, MA, USA; Stacie G. Deiner MD Department of Anesthesiology, The Icahn School of Medicine at Mount Sinai, New York, NY, USA; Diederik van Dijk MD PhD University Medical Center Utrecht, Utrecht, The Netherlands; Steven T. DeKosky MD McKnight Brain Institute, Department of Neurology, College of Medicine, University of Florida, Gainesville FL, USA; Maryellen F. Eckenhoff PhD Department of Anesthesiology and Critical Care University of Pennsylvania School of Medicine Philadelphia, PA, USA; Lars Eriksson MD PhD Section for Anesthesiology and Intensive Care Medicine Karolinska Institutet and Karolinska University Hospital, Stockholm, Sweden; Dougas Galasko MD Shiley-Marcos Alzheimer's Disease Research Center and Department of Neurosciences, University of California, San Diego, CA, USA; Kirk Hogan MD JD Department of Anesthesiology, University of Wisconsin, WI, USA; Sharon Inouye MD MPH Beth Israel Deaconess Medical Center, Harvard Medical School, Aging Brain Center, Institute for Aging Research, Hebrew SeniorLife, Boston, MA, USA; David Knopman MD Department of Neurology, Mayo Clinic, Rochester, MN, USA; Constantine Lyketsos MD PhD Department of Psychiatry and Behavioral Sciences, Johns Hopkins Medicine, Johns Hopkins University, Baltimore, MD, USA; Edward Marcantonio MD Harvard Medical School, Division of General Medicine and Primary Care, Beth Israel Deaconess Medical Center, Boston, MA, USA; Paul Maruff PhD Professor, Centre for Neuroscience, University of Melbourne. Melbourne, Australia; Mervyn Maze MD Department of Anaesthesia and Perioperative Care, UCSF \& Center for Cerebrovascular Research Zuckerberg San Francisco General, San Francisco, CA, USA; Esther Oh MD PhD Division of Geriatric Medicine and Gerontology, the Johns Hopkins University School of Medicine, Balt, MD, USA; Beverley A. Orser MD PhD University of Toronto, Department of Anaesthesia, Sunnybrook Health Science Centre, Toronto, Canada; Thomas Ottens MD PhD Department of Anesthesiology,
University Medical Center Utrecht, Utrecht, The Netherlands; Catherine Price PhD Clinical \& Health Psychology, University of Florida College of Public Health and Health Professions, Gainesville, FL, USA; Lars S. Rasmussen MD PhD Department of Anaesthesia, Center of Head and Orthopaedics, Rigshospitalet, University of Copenhagen, Denmark; Perminder Sachdev MD PhD Centre for Healthy Brain Ageing (CHeBA), School of Psychiatry, UNSW Medicine \& Neuropsychiatric Institute, The Prince of Wales Hospital, Sydney, Australia; Katie Schenning MD MPH Department of Anesthesiology \& Perioperative Medicine, Oregon Health \& Science University, Portland, OR, USA; David A. Scott MB BS PhD St. Vincent's 
Hospital Melbourne \& University of Melbourne, Australia; Frederick E. Seiber MD Department of Anaesthesia/Critical Care Medicine, Johns Hopkins Bayview Medical Center, Johns Hopkins Medical Institutions, Baltimore, MD, USA; Brendan Silbert MB BS St. Vincent's Hospital Melbourne \& University of Melbourne, Australia; Jeff Silverstein MD Department of Anesthesiology, Mount Sinai School of Medicine, New York, NY, USA; Jacob Steinmetz MD PhD Department of Anaesthesia, Centre of Head and Orthopaedics, Rigshospitalet, University of Copenhagen, Denmark; Niccolo Terrando BSc (Hons) DIC PhD Department of Anesthesiology, Duke University Medical Center, Durham, NC, USA; Paula Trzapacz MD Indiana University School of Medicine, Indianapolis, IN, USA; Rob Whittington MD Columbia University College of Physicians and Surgeons, New York, NY, USA; Zhongcong Xie MD PhD Harvard Medical School, Department of Anaesthesia, Critical Care and Pain Medicine, Massachusetts General Hospital, Charlestown, MA, USA.

Five contributors from the working party (not listed) elected not to be included as authors, each for a different reason.

\section{References}

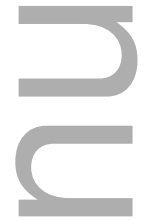

1. Moller JT, Cluitmans P, Rasmussen LS, et al. Long-term postoperative cognitive dysfunction in the elderly ISPOCD1 study. ISPOCD investigators. International Study of Post-Operative Cognitive Dysfunction. Lancet 1998; 351: 857 e61

2. Evered L, Scott DA, Silbert B, Maruff P. Postoperative cognitive dysfunction is independent of type of surgery and anesthetic. Anesth Analg 2011; 112: 1179e85

3. Mathew JP, Mackensen GB, Phillips-Bute B, et al. Randomized, double-blinded, placebo controlled study of neuroprotection with lidocaine in cardiac surgery. Stroke 2009; 40: 880e7

4. Evered LA, Silbert BS, Scott DA, Maruff P, Ames D. Prevalence of dementia 7.5 years after coronary artery bypass graft surgery. Anesthesiology 2016; 125: 62e71

5. Monk TG, Saini V, Weldon BC, Sigl JC. Anesthetic management and one-year mortality after noncardiac surgery. Anesth Analg 2005; 100: 4e10

6. Lewis MS, Maruff P, Silbert BS, Evered LA, Scott DA. Detection of postoperative cognitive decline after coronary artery bypass graft surgery is affected by the number of neuropsychological tests in the assessment battery. Ann Thorac Surg 2006; 81: 2097e104

7. Australian Government. Australian hospital statistics 2007e08. Canberra: Australian Institute of Health and Welfare; 2009

8. Number of procedures UAf. 2012. Available from: http:// www.cdc.gov/nchs/data/nhds/4procedures/2010pro4 numberprocedureage.pdf [Accessed 1 June 2017]

9. American Psychiatric Association (APA). Diagnostic and statistical manual of mental disorders. 5th ed. Arlington, VA, USA: American Psychiatric Publishing; 2013

10. Petersen RC, Roberts RO, Knopman DS, et al. Mild cognitive impairment: ten years later. Arch Neurol 2009; 66: 1447e55

11. Australian Institute of Health and Welfare (AIHW). Dementia in Australia. Canberra: AIHW; 2012

12. Boulkedid R, Abdoul H, Loustau M, Sibony O, Alberti C. Using and reporting the Delphi method for selecting healthcare quality indicators: a systematic review. PLoS One 2011; 6: e20476 
13. Albert MS, DeKosky ST, Dickson D, et al. The diagnosis of mild cognitive impairment due to Alzheimer's disease: recommendations from the National Institute on Aging eAlzheimer's Association workgroups on diagnostic guidelines for Alzheimer's disease. Alzheimers Dement 2011; 7: 270e9

14. McKhann GM, Knopman DS, Chertkow H, et al. The diagnosis of dementia due to Alzheimer's disease: recommendations from the National Institute on Aging eAlzheimer's Association workgroups on diagnostic guidelines for Alzheimer's disease. Alzheimers Dement 2011; 7: 263e9

15. Rudolph JL, Marcantonio ER. Review articles: postoperative delirium: acute change with long-term implications. Anesth Analg 2011; 112: 1202e11

16. Newman MF, Kirchner JL, Phillips-Bute B, et al. Longitudinal assessment of neurocognitive function after coronary-artery bypass surgery. N Engl J Med 2001; 344: $395 e 402$

17. Inouye SK, Marcantonio ER, Kosar CM, et al. The shortterm and long-term relationship between delirium and cognitive trajectory in older surgical patients. Alzheimers Dement 2016; 12: 766 e75

18. Murkin JM, Newman SP, Stump DA, Blumenthal JA. Statement of consensus on assessment of neurobehavioral outcomes after cardiac surgery. Ann Thorac Surg 1995; 59: 1289e95
19. Selnes OA, Royall RM, Grega MA, Borowicz Jr LM, Quaskey S, McKhann GM. Cognitive changes 5 years after coronary artery bypass grafting: is there evidence of late decline? Arch Neurol 2001; 58: 598e604

20. Lewis MS, Maruff P, Silbert BS, Evered LA, Scott DA. The influence of different error estimates in the detection of post-operative cognitive dysfunction using reliable change indices with correction for practice effects. Arch Clin Neuropsychol 2006; 21: 421 e7

21. Rasmussen LS, Larsen K, Houx P, Skovgaard LT, Hanning CD, Moller JT. The assessment of postoperative cognitive function. Acta Anaesth Scand 2001; 45: 275 e89

22. Schneider LS, Clark CM, Doody R, et al. ADCS Prevention Instrument Project: ADCS-clinicians' global impression of change scales (ADCS-CGIC), self-rated and study partnerrated versions. Alzheimer Dis Assoc Disord 2006; 20: S124e 38

23. Hogue Jr CW, Hershey T, Dixon D, et al. Preexisting cognitive impairment in women before cardiac surgery and its relationship with $\mathrm{C}$-reactive protein concentrations. Anesth Analg 2006; 102: 1602e8

24. Evered LA, Silbert BS, Scott DA, Maruff P, Ames D, Choong PF. Preexisting cognitive impairment and mild cognitive impairment in subjects presenting for total hip joint replacement. Anesthesiology 2011; 114: 1297e304

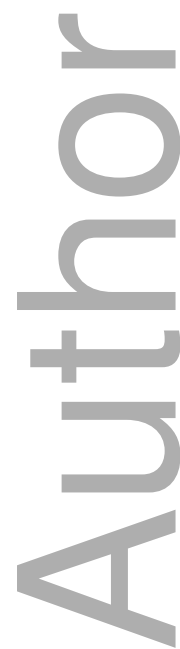

Handling editor: H.C. Hemmings Jr 


\section{University Library}

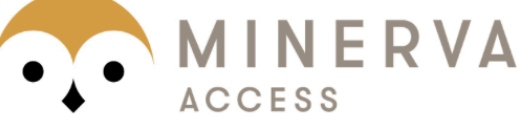

A gateway to Melbourne's research publications

Minerva Access is the Institutional Repository of The University of Melbourne

Author/s:

Evered, L;Silbert, B;Knopman, DS;Scott, DA;DeKosky, ST;Rasmussen, LS;Oh, ES;Crosby, G;Berger, M;Eckenhoff, RG

Title:

Recommendations for the nomenclature of cognitive change associated with anaesthesia and surgery-2018

Date:

2018-11-01

Citation:

Evered, L., Silbert, B., Knopman, D. S., Scott, D. A., DeKosky, S. T., Rasmussen, L. S., Oh, E. S., Crosby, G., Berger, M. \& Eckenhoff, R. G. (2018). Recommendations for the nomenclature of cognitive change associated with anaesthesia and surgery-2018. ACTA ANAESTHESIOLOGICA SCANDINAVICA, 62 (10), pp.1473-1480. https://doi.org/10.1111/ aas. 13250.

Persistent Link:

http://hdl.handle.net/11343/284639 